\title{
Le rythme sinusal
}

\section{Mécanisme et fonction}

Guy Lerebours

$>$ Le rythme cardiaque normal est commandé par une formation spécifique située dans l'oreillette droite: le nœud sinusal. Le noeud sinusal est doué d'automatisme et peut engendrer spontanément, grâce à des propriétés électriques bien identifiées, des impulsions qui sont à la source du battement cardiaque. La fréquence de production des impulsions détermine celle du rythme normal dit sinusal. En fait, l'activité propre du nœud sinusal est en permanence modulée par des neurotransmetteurs émanant de systèmes cholinergiques et adrénergiques. De telles influences s'exercent en permanence par le jeu d'interactions complexes dont l'effet résultant sur le nœud sinusal produit la fréquence cardiaque observée. La modulation nerveuse a pour effet d'adapter la fréquence cardiaque, et par conséquent le débit sanguin, aux besoins métaboliques des tissus. Elle constitue un facteur d'homéostasie. Si la bradycardie du sommeil est associée à une forme d'hibernation, à l'opposé l'activité diurne et l'effort, en stimulant la libération de catécholamines, produisent une augmentation salutaire de la fréquence cardiaque. Le rythme sinusal normal est aussi un déterminant essentiel de la qualité de vie. <

Chez l'être vivant, la circulation du sang nécessaire au fonctionnement des tissus auxquels elle apporte oxygène et nutriments et dont elle assure l'évacuation des déchets, est produite par les contractions rythmées du cœur. Chaque contraction, en réduisant le volume des cavités ventriculaires, entraîne l'éjection du sang dans le système vasculaire. Travaillant de manière synchronisée, le ventricule droit chasse le sang veineux dans la circulation pulmonaire où celui-ci va s'oxygéner tandis que le ventricule gauche envoie le sang rouge oxygéné dans l'aorte et les artères périphériques. Dans les artères, chaque cycle d'activité cardiaque est responsable d'une onde pulsatile dont on peut détecter la présence en prenant le

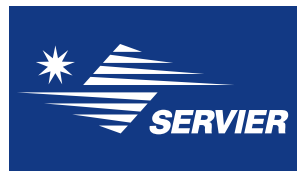

Institut de recherches internationales Servier, 6, place des Pléiades, 92415 Courbevoie, France.

pouls. Par ailleurs, lors de la contraction cardiaque, le sang est d'abord retenu sous pression dans les ventricules avant que le barrage à la sortie ne cède puis se referme à nouveau. Ces événements mettent en cause des valvules qui, en se fermant, causent les bruits cardiaques entendus à l'auscultation. Le rythme cardiaque peut donc être appréhendé manuellement par le pouls et de manière auditive par le stéthoscope. L'électrocardiogramme enregistre l'activité électrique du cœur marquée par une succession de complexes électriques constitués de plusieurs ondes. La fonction contractile du myocarde est liée aux propriétés électrophysiologiques des cellules cardiaques: en effet, l'activité électrique précède et déclenche l'activité contractile de la cellule cardiaque, il s'agit du couplage excitation-contraction.

\section{Le tissu spécialisé ou tissu nodal}

L'essentiel du tissu cardiaque est constitué de cardiomyocytes. Ce sont des cellules musculaires qui possèdent un appareil myofibrillaire et douées de propriété contractile. Les cardiomyocytes peuplent les parois cardiaques et les plus gros contingents colonisent les ventricules, tout particulièrement le ventricule gauche. Ces cellules musculaires se distinguent de celles des muscles squelettiques par leur propriété de rester, après chaque contraction, inexcitable: cette période réfractaire prévient la tétanisation.

Le myocarde possède aussi des formations de structure histologique distincte appelée tissu spécialisé de conduction: tissu nodal et système His-Purkinje. Les cellules qui les composent ont un aspect embryonnaire avec des constituants myofibrillaires peu évolués. Une première formation est présente à la partie haute de l'oreillette droite: il s'agit du nœud sinusal. Il est situé dans l'oreillette droite au niveau de la jonction avec la veine cave supérieure et près de la crista terminalis. Chez l'homme, sa longueur varie de 7 à $20 \mathrm{~mm}$ et sa largeur de 2 à $5 \mathrm{~mm}$. 
Dans le pannicule graisseux à son contact, existent des ganglions nerveux en nombre qui sont responsables d'influences neurogènes sur l'activité sinusale. Plus bas, on retrouve une autre formation située dans la partie haute du septum interventriculaire membraneux au niveau du trigone : le nœud auriculo-ventriculaire qui joue le rôle de filtre entre l'activité auriculaire et ventriculaire. Puis le faisceau de His dont chaque branche de division s'engage dans le ventricule correspondant et s'arborise en de multiples ramifications, le réseau de Purkinje, tissu de jonction avec les fibres myocardiques (Figure 1).

Les études de l'activité électrique cardiaque, expérimentales et cliniques, ont montré qu'au départ d'un battement il y a émergence d'une primo-dépolarisation dans la région du nœud sinusal [1]. Puis une onde d'excitation se propage le long du tissu nodal d'abord dans les oreillettes vers le nœud auriculo-ventriculaire de Tawara, avant d'être acheminée par le faisceau de His et ses branches jusqu'au contact du muscle ventriculaire qui se dépolarise à son tour. Les temps de conduction et les vitesses de conduction varient le long du tissu nodal : extrêmement lente dans le nœud sinusal, lente dans le nœud auriculo-ventriculaire afin d'assurer le remplissage ventriculaire, la vitesse de conduction est à l'inverse très rapide dans le faisceau de His et le réseau de Purkinje afin de dépolariser rapidement l'ensemble des myocytes de façon syncitiale et de synchroniser la contraction des deux ventricules.. Le phénomène se reproduit ensuite à une cadence donnée, laquelle se chiffre en battements par minute. Au sein du tissu spécialisé, le nœud sinusal est le générateur d'impulsions, chacune engendrant une dépolarisation cardiaque (pacemaker). Cette activité est automatique, c'est-à-dire qu'elle détient en elle-même son propre mode de déclenchement. Aucun agent extérieur n'est requis comme le démontre le fait qu'expérimentalement, un cœur excisé, placé dans un milieu oxygéné, peut continuer de battre spontanément.

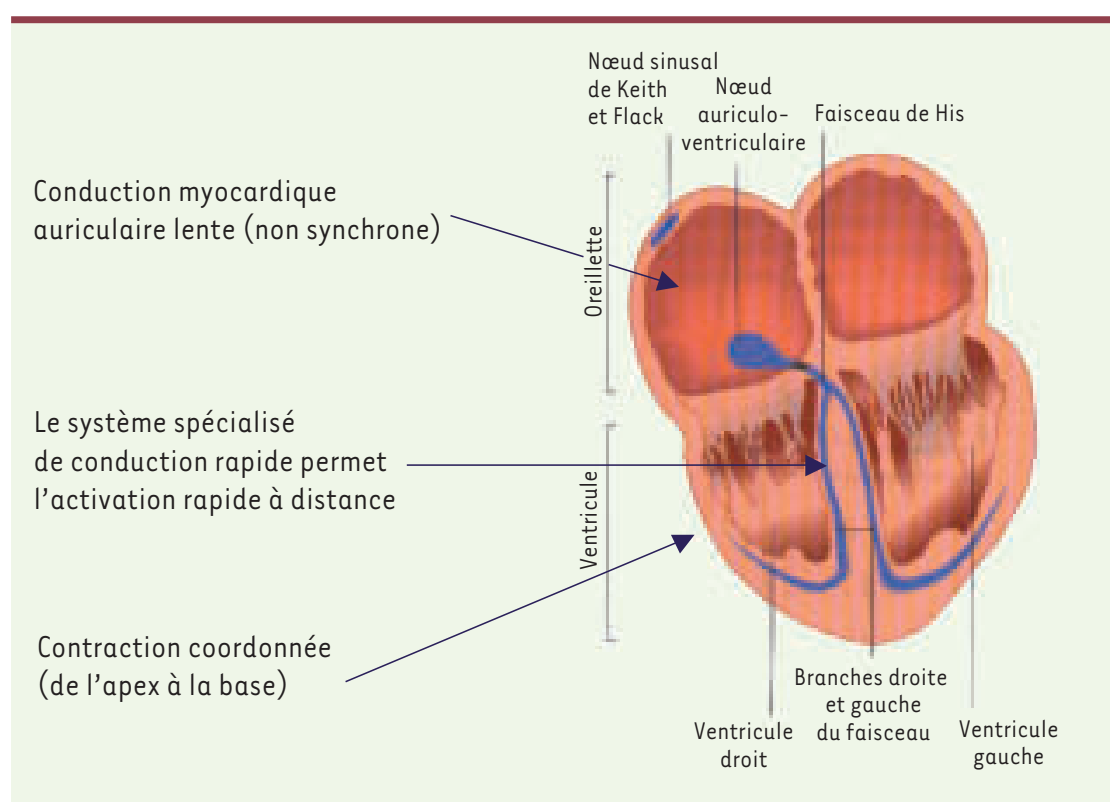

Figure 1. Le tissu cardiaque.

\section{Nœud sinusal : genèse de l'automatisme cardiaque}

La production d'une impulsion électrique s'effectue au sein d'îlots cellulaires dominants situés au centre du nœud qui constituent la source de cette activité. Le processus se répand ensuite dans le reste du nœud sinusal et le tissu myocardique voisin. La production automatique et rythmée d'impulsions a pu être expliquée grâce à l'introduction de microélectrodes dans les cellules sinusales [1]. Cette technique permet l'enregistrement du potentiel transmembranaire: il s'agit de la différence de potentiel électrique mesurée entre les 2 faces de la membrane. L'activation ou dépolarisation des cellules sinusales entraîne une variation rapide de potentiel de l'ordre de 70 à $80 \mathrm{mV}$ suivie d'un retour lent à l'état diastolique initial: un potentiel d'action est activé. Les cellules voisines, réagissant à cette impulsion, produisent à leur tour un nouveau potentiel d'action, et ainsi, de proche en proche, la vague dépolarisante s'étend à l'ensemble du cœur (Figure 2).

La succession de potentiels d'action formés dans le nœud sinusal assure l'automatisme cardiaque. Ce phénomène a été étudié à l'aide des enregistrements intracellulaires [2]. Ainsi, il a été observé qu'après chaque battement, le potentiel électrique diastolique de repos, qui est revenu autour de $-60 \mathrm{mV}$, n'est pas constant et évolue vers des valeurs de potentiel membranaire plus positive (pente de dépolarisation diastolique) jusqu'à atteindre le seuil d'activation du potentiel d'action. En d'autres termes, la cellule sinusale a la capacité d'abaisser d'elle-même son potentiel de repos jusqu'à un niveau correspondant au seuil de déclenchement d'une impulsion propagée (Figure 3). La cellule sinusale fait spontanément ce qui, dans tout tissu excitable, requiert l'intervention d'un agent extérieur, nerveux ou humoral. Cette dépolarisation diastolique lente est caractéristique des cellules responsables de l'automaticité cardiaque. On l'explique par le jeu de courants ioniques transmembranaires dépolarisants et repolarisants. L'effet net est un enrichissement progressif de la cellule en charges positives, d'où le processus de dépolarisation [3, 4].

Plusieurs courants sont principalement impliqués dans cette phase (Figure 4): le courant pacemaker $I_{\text {f }}$, les courants calciques $I_{\text {CaL }}$ et $I_{\text {CaT }}$, le courant de fond $I_{\text {CaNa }}$, le courant $I_{\mathrm{Kr}}$. 


\section{- Courant $I_{f}$}

Le courant $I_{f}$ est un courant mixte porté par les ions sodium et potassium. Cependant le courant principal est dû aux ions $\mathrm{Na}^{+}$qui ont une plus grande perméabilité que les ions $\mathrm{K}^{+}$. Son $\mathrm{V}_{\text {inv }}$ est de $-20 \mathrm{mV}$. En conséquence, $I_{\mathrm{f}}$ est un courant entrant durant la dépolarisation diastolique et devient sortant durant la dépolarisation rapide et la phase de repolarisation du potentiel d'action. II est activé par l'hyperpolarisation membranaire à partir de -60 à $-40 \mathrm{mV}$ à la fin de la phase de repolarisation. II initie la dépolarisation diastolique et contribue aux recrutements des autres canaux voltage-dépendants qui sont activés par la dépolarisation (canaux calciques $\mathrm{T}$ et $\mathrm{L}$ ).

\section{- Courant potassique retardé $\left(I_{\mathrm{Kr}}\right)$}

L'activation de ce courant potassique concourt de façon importante à la phase de repolarisation alors que sa désactivation est nécessaire à la dépolarisation diastolique spontanée.

\section{- Courants calciques $\left(/_{\text {CaL }}, /_{\text {CaT }}\right)$}

Les canaux calciques de type $L$ contribuent à la phase finale de la dépolarisation diastolique et au potentiel d'action.

Le rôle des canaux calciques de type T est moins connu et semble limité à la dépolarisation diastolique sans aucun effet sur le potentiel d'action.

\section{- Autres courants}

D'autres courants participent à la modulation du potentiel diastolique: il s'agit du courant de fond entrant sodique, du courant entrant d'échange $\mathrm{Na} / \mathrm{Ca}$.

La cadence sinusale est dépendante de la pente de dépolarisation diastolique. Plus elle est rapide, c'est

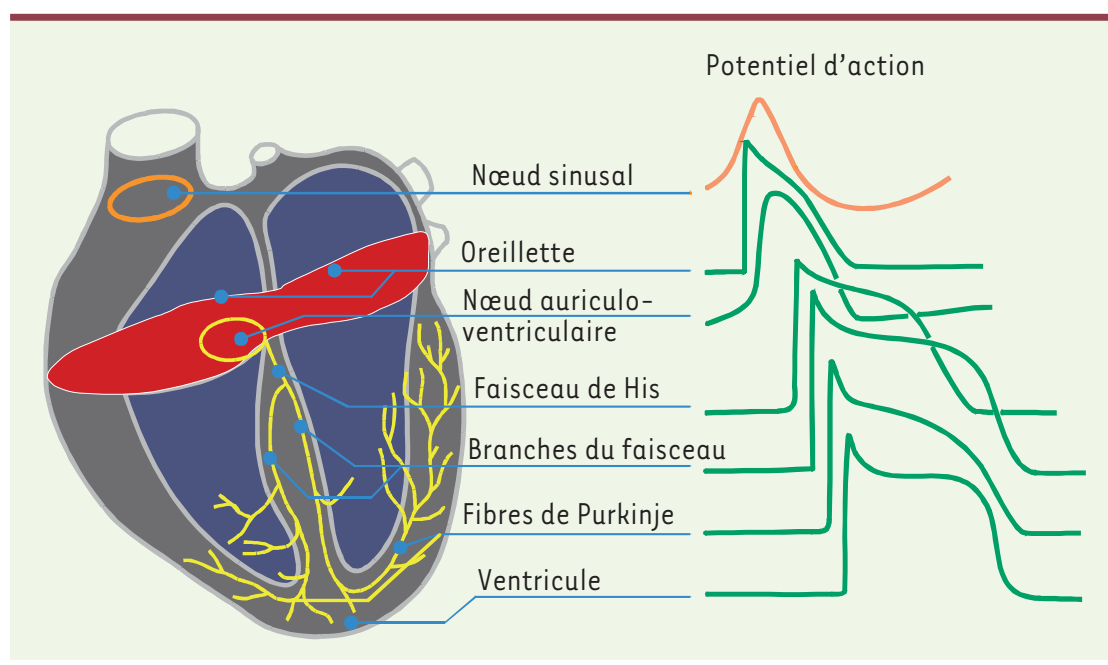

Figure 2. Nœud sinusal : genèse de l'automatisme cardiaque. le cas par exemple quand la température s'élève, plus le rythme sinusal est rapide. À l'opposé, tout ralentissement de la dépolarisation diastolique s'accompagne de diminution de la fréquence cardiaque. On verra par la suite que diverses influences neuro-hormonales sur le rythme cardiaque s'exercent principalement en modifiant la pente de dépolarisation diastolique spontanée. À cela s'ajoutent des effets éventuels sur le potentiel diastolique de départ ou sur le potentielseuil, également susceptibles de modifier le temps de passage de l'un à l'autre.

L'activité sinusale domine l'automatisme d'autres foyers plus périphériques. Ces derniers sont envahis par l'onde de dépolarisation avant d'avoir pu s'exprimer. En revanche, en cas d'insuffisance du foyer dominant (maladie du sinus), la formation de l'impulsion cardiaque est assurée par ces pacemakers de substitution [5]. La source électrique est déplacée vers les régions basses du nœud sinusal. Sur l'électrocardiogramme s'inscrivent des ondes P modifiées, témoins de la migration du pacemaker sinusal (wandering pacemaker).

\section{Rôle du système nerveux autonome}

Grâce au système nerveux autonome, le cœur peut moduler son activité en fonction d'états physiologiques variés et aussi d'événements anormaux. Le nœud sinusal est richement innervé. II existe deux types de terminaisons nerveuses: sympathiques et parasympathiques d'où sont libérés les neurotransmetteurs qui se fixent sur des récepteurs de la membrane de la cellule cardiaque. Un processus de contrôle réciproque intervient constamment pour amortir et corriger les à-coups de l'un ou l'autre système. L'activité parasympathique de fond encore appelée tonus vagal, est associée à un abaissement de la fréquence cardiaque. L'acétylcholine en est le médiateur. Le système cholinergique est lui-même sollicité par toute augmentation de la pression sanguine au niveau de barorécepteurs situés dans la crosse aortique et le sinus carotidien. La cellule sinusale montre une diminution de la pente de dépolarisation diastolique spontanée qui, en conjonction avec un potentiel de repos plus électronégatif, concourt à en ralentir la décharge (Figure 1) [7]. Une augmentation du tonus vagal, en déprimant préférentiellement les zones d'automatisme dominant, peut contribuer à l'émergence de rythmes parasinusaux d'échappement voire d'autres plus lents nés dans la jonction auriculo-ventriculaire (rythmes jonctionnels). À l'extrême, une décharge parasympathique excessive peut inhiber durablement l'activité automatique où qu'elle soit, engendrant une pause cardiaque plus ou moins prolongée pouvant conduire à une syncope dite vagale. 


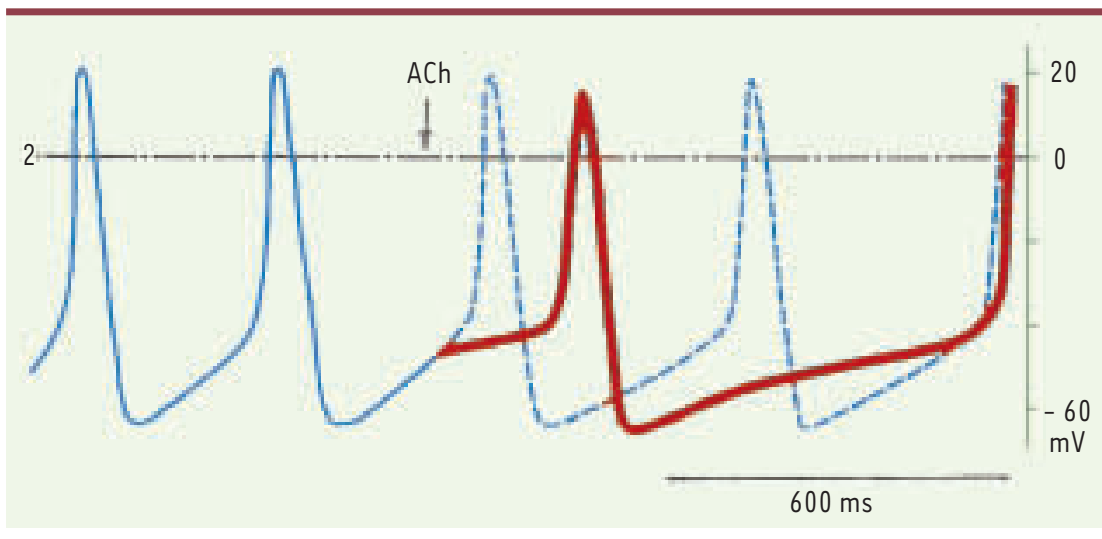

Figure 3. Potentiels d'action de cellules sinusales fournis par des enregistrements intracellulaires. On voit que le potentiel diastolique maximum mesure autour de $-60 \mathrm{mV}$. Lui fait suite une diminution progressive du voltage jusqu'à - $40 \mathrm{mV}$, valeur du potentiel seuil. Cette dépolarisation diastolique lente spontanée est le support de l'automatisme sinusal. Une fois le seuil atteint, une soudaine variation de potentiel de $-40 \grave{a}+20 \mathrm{mV}$ a lieu. Le potentiel d'action ainsi produit contribue à propager le phénomène électrique. À la partie droite de la figure, sont montrés les effets de la libération d'acétylcholine (ACh) lors de la stimulation vagale. Les potentiels d'action en pointillé figurent ceux qui se seraient maintenus en l'absence d'action cholinergique. La libération d'acétylcholine entraîne des modifications figurées en trait gras. Le potentiel diastolique maximum devient plus électronégatif (hyperpolarisation). Surtout la dépolarisation diastolique spontanée est nettement ralentie. Le tout contribue à diminuer la fréquence de décharge des cellules sinusales.

L'effet du tonus sympathique s'exerce par l'intermédiaire des catécholamines, libérés par les terminaisons nerveuses, mais aussi, par la glande médullosurrénale. En réponse à la stimulation adrénergique via les récepteurs $\beta$, le rythme sinusal s'accélère. Les études in vitro de cel-

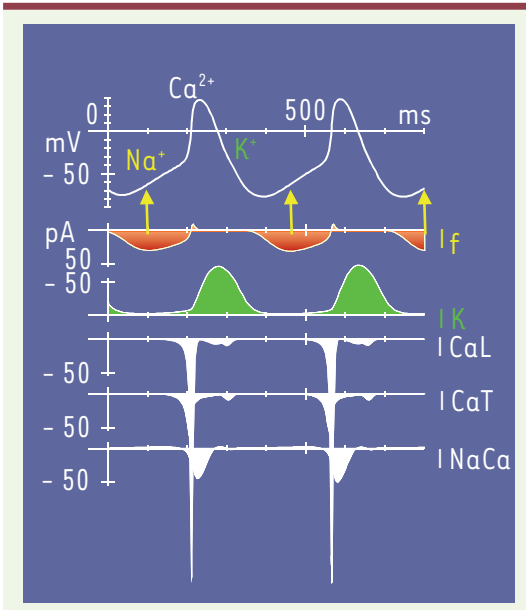

Figure 4. Simulation informatisée des différents courants ioniques transmembranaires dépolarisants et repolarisants dans une cellule de nœud sinusal. Le courant $I_{f}$ est celui qui contribue le plus à la dépolarisation diastolique hormis dans sa phase tardive (d'après [16]). aux exigences du métabolisme tissulaire: apport en oxygène et en substances nutritives aux tissus utilisateurs. L'intervention du système adrénergique répond à toute augmentation de l'activité métabolique, comme en produit typiquement l'exercice physique. Le tonus vagal pour sa part assure le retour à l'équilibre et, lorsqu'il prédomine, maintient le fonctionnement tissulaire en régime d'économie.

\section{Caractéristiques du rythme cardiaque normal}

Ainsi le rythme cardiaque normal est un rythme sinusal. La dépolarisation qui succède à l'impulsion sinusale et affecte successivement oreillettes et ventricules se traduit sur l'électrocardiogramme par la succession d'une onde $P$ et d'un complexe QRS avec entre les deux un espace, l'intervalle PR, témoin du passage de la jonction auriculo-ventriculaire [9]. La fréquence habituelle des battements cardiaques au repos est de l'ordre de 60 à 80 par minute. Elle reflète la cadence de décharge des cellules sinusales, mais en partie seulement. En effet, le système nerveux autonome joue aussi un rôle, et plus particulièrement sa composante cholinergique, laquelle, en l'absence d'effort, voit ses effets l'emporter sur les influences sympathiques et contribuer de manière prédominante au contrôle de la fréquence sinusale [5]. Le blocage pharmacologique momentané du contrôle nerveux par l'administration conjointe d'agents $\beta$-bloquants et anticholinergiques démasque la véritable fréquence sinusale intrinsèque, toujours plus élevée que celle observée et qui s'abaisse avec l'âge [10].

Des fluctuations de la fréquence sinusale de courte période sont imbriquées dans d'autres qui s'étirent sur des temps prolongés, telle l'alternance de rythmes nocturnes lents et diurnes plus rapides. Cette variabilité sinusale tient au jeu d'interactions complexes entre les composantes adrénergiques et cholinergiques du système nerveux autonome [11]. Les mouvements respiratoires (inspiration/expiration), surtout perceptibles chez le sujet jeune, sont responsables de succession de phases d'accélération et de ralentissement du rythme cardiaque, d'où le nom d'arythmie respiratoire (Figure 2). 
Des fréquences sinusales au-dessous de 50 battements par minute, ou bradycardies, ont toute chance de traduire une prépondérance du tonus vagal. Celle-ci peut être momentanée comme c'est le cas des rythmes lents se produisant pendant le sommeil. Des pauses sinusales de 3 secondes et plus sont éventuellement détectables par des enregistrements continus ambulatoires de l'électrocardiogramme par la technique dite de Holter. La phase nocturne du nycthémère s'associe en effet à une hypervagotonie. Cette hypervagotonie peut aussi constituer un état permanent chez certains sujets. On invoque alors une notion de terrain, voire un contexte familial. La bradycardie sinusale de fond a pour corollaire des fréquences d'effort modérées. Il y a de ce fait économie d'énergie, une condition favorable à l'exercice du sport et à l'en-

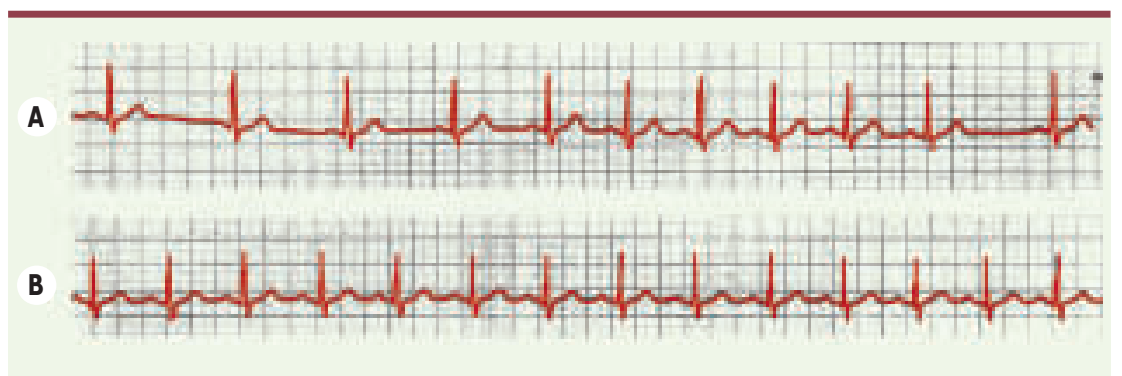

Figure 5. Arythmie respiratoire du sujet jeune. L'électrocardiogramme du haut (tracé $\boldsymbol{A}$ ) montre la succession de battements sinusaux. L'irrégularité du rythme cardiaque est manifeste avec des phases plus rapides (battements du milieu) et d'autres ralenties (aux deux extrémités du tracé). Cette alternance est dépendante des mouvements respiratoires. La confirmation en est fournie par le tracé du bas (B). Alors que le sujet a arrêté quelques instants sa respiration, le rythme est simultanément devenu régulier, avec une cadence similaire aux phases rapides précédentes. À l'évidence a été levée l'action inhibitrice du tonus vagal stimulée par la respiration.

\begin{tabular}{lccc}
\hline & $\beta$-adrénergique & $\boldsymbol{\alpha}$-adrénergique & cholinergique \\
$\begin{array}{l}\text { Nœud sinusal } \\
\begin{array}{l}\text { Conduction } \\
\text { dans le nœud A-V }\end{array}\end{array}$ & + & 0 & - \\
$\begin{array}{l}\text { Conduction } \\
\text { dans le système HP }\end{array}$ & + & 0 & - \\
$\begin{array}{l}\text { Contraction } \\
\text { myocardique }\end{array}$ & + & 0 & - \\
\hline
\end{tabular}

Tableau I. Effets opposés de la stimulation adrénergique et cholinergique sur le cœur. L'effet d'augmentation de l'automatisme sinusal, de la conduction auriculo-ventriculaire et de la contractilité myocardique dû à la stimulation adrénergique fait intervenir les seuls récepteurs $\beta$. La stimulation cholinergique exerce en tous points une action antagoniste. A-V : auriculo-ventriculaire; HP : His-Purkinje. durance. Chez les sportifs de haut niveau, l'entraînement intensif renforce la prépondérance du tonus vagal II peut en résulter sur l'électrocardiogramme, outre une bradycardie sinusale marquée, un allongement de l'intervalle PR, témoin du freinage vagal de la conduction auriculo-ventriculaire $[12,13]$.

dessus de 100 battements par minute, on parle de tachycardiverses, physiques ou émotionnelles, la réaction nerveuse visant à une réponse adaptée. Au cours d'un effort, apparaissent dans areil musculaire des besoins en oxygène et nutriments qui peuvent être considérables. Pour les satisfaire, le cœur augmente la fréquence de ses battements [14]. Lors de tests d'effort sur tation corrélative de la fréquence cardiaque qui peut atteindre et - l'âge du sujet). Pour le diagnostic de troubles coronariens, on s'en tient habituellement à $80 \%$ de la fréquence maximale théorique (encore compatible avec le maintien en aérobiose).

\section{Conclusion : aspects physiopathologiques}

Engendré par l'automatisme sinusal, le rythme cardiaque normal est sous le contrôle des neurotransmetteurs adrénergiques et cholinergiques. Sur le plan hémodynamique, le battement résultant trouve sa pleine efficacité dans la mesure où est réalisée, selon un délai approprié, la succession d'une contraction auriculaire puis ventriculaire, la première parachevant le remplissage des ventricules et en optimisant ainsi la performance. Des mécanismes anormaux peuvent entrer en jeu qui donnent lieu à des troubles cliniques. L'origine de l'impulsion cardiaque est en la circonstance déplacée du nœud sinusal vers des sites ectopiques dans les oreillettes, la jonction auriculo-ventriculaire ou les ventricules. Lorsque leur cadence est suffisamment élevée, de tels rythmes prennent alors la commande. C'est le cas des tachycardies paroxystiques observées en clinique. En l'occurrence, le phénomène évolue pour son propre compte et échappe aux mécanismes de contrôle du rythme sinusal. Ce dernier, après correction du désordre, peut redémarrer lentement et mettre un certain temps à récupérer sa fréquence propre: on parle d'inhibition post-stimulation [15]. Comparé aux événements anormaux qui 
sont susceptibles d'en troubler le cours, le rythme sinusal demeure celui qui confère au sujet les aptitudes physiques les plus élevées et la meilleure qualité de vie. $\diamond$

\section{SUMMARY}

Sinus rhythm: mechanisms and function

The normal cardiac rhythm originates in a specialized region of the heart, the sinus node that is part of the nodal tissue. The rhythmic, impulse initiation of sinus node pacemaker cells results from a spontaneous diastolic depolarization that is initiated immediately after repolarization of the preceding actions potential. This slow diastolic depolarisation is typical of automatic cells and essential to their function. Several currents are involved in this diastolic depolarisation: a hyperpolarisation activated inward current, termed "pacemaker" If current, two $\mathrm{Ca}^{2+}$ currents (a $L$ type and a $\mathrm{T}$ type), a delayed $\mathrm{K}^{+}$current and a $\mathrm{Na} / \mathrm{Ca}$ exchange current. The frequency of the automatic discharge is the main determinant of heart rate. However the sinus node activity is regulated by adrenergic and cholinergic neurotransmitters. Acetylcholine provokes the hyperpolarisation of pacemaker cells and decreases the speed of the spontaneous diastolic depolarisation, thus slowing the sinus rate. Catecholamines lead to sinus tachycardia by increasing the diastolic depolarisation speed. In normal conditions, the observed resting heart rate is lower than the intrinsic frequency of the sinus node due to a "predominance" of the vagal tone. Neural regulation of the heart rate aims at meeting the metabolic needs of the tissues through a varying blood flow. Differences between diurnal and nocturnal mean heart rates are accounted for by neural influences. During the night, the increased vagal tone results in decreased heart rate. The exercise-induced tachycardia results from the sympathetic stimulation. It allows more blood to reach skeletal muscles, and as a consequence an increased supply of oxygen and nutrients. Compared to the variety of clinical arrhythmias, sinus rhythm is the basis for optimal exercise capacity and quality of life. $\diamond$

\section{REMERCIEMENTS}

Je tiens à remercier le Professeur Samuel Levy, Hôpital Nord, Service de Cardiologie, 13015 Marseille, France, pour la relecture critique de cet article.

\section{RÉFÉRENCES}

1. Brown HF. Electrophysiology of the sinoatrial node. Physiol Rev 1982 ; $62: 505-30$.

2. Brown HF, DiFrancesco D. Voltage-clamp investigations of membrane currents underlying pacemaker activity in rabbit sino-atrial node. J Physiol $1980 ; 308: 331-51$.

3. Brown HF, Kimura J, Noble D, et al. The ionic currents underlying pacemaker activity in rabbit sino-atrial node: experimental results and computer simulations. Proc R Soc Lond 1984 ; 222 : 329-47.

4. Oei HI, Van Ginneken ACG, Jongsma HJ, Bouman LN. Mechanisms of impulse generation in isolated cells from the rabbit sinoatrial node. J Mol Cell Cardiol $1989 ; 21: 1137-49$

5. Michaels DC, Matyas EP, Jalife J. Mechanisms of sinoatrial pacemaker synchronization : a new hypothesis. Circ Res 1987 ; 61 : 704-12.

6. Opthof T. The mammalian sinoatrial node. Cardiovasc Drugs Ther 1988 ; $1: 573-97$.

7. Trautwein W, Kuffler SW, Edwards C. Changes in membrane characteristics of heart muscle during inhibition. J Gen Physiol 1956; 40 : 135-45.

8. Hagiwara N, Irisawa H, Kameyama M. Contribution of two types of calcium currents to the pacemaker potentials of rabbit sinoatrial node cells. J Physiol 1988 ; $395: 233-53$.

9. Scher AM, Rodriguez MI, Liikane J, Young AC. The mechanism of atrioventricular conduction. Circ Res $1959 ; 7: 357-92$.

10. Jose AD. Effect of combined sympathetic and parasympathetic blockade on heart rate and cardiac function in man. Am J Cardiol 1966 ; $18: 476-8$.

11. Akselrod S, Gordon D, Ubel FA, et al. Power spectrum analysis of heart rate fluctuations: a quantitative probe of beat-to-beat cardiovascular control. Science $1981 ; 213: 220-2$.

12. Huston TP, Puffer JC, Rodney WN. The athletic heart syndrome. $N$ EnglJ Med $1985 ; 313: 24-32$.

13. Oakley CM. The electrocardiogram in the highly trained athlete. Cardio Clin $1992 ; 10: 295-302$.

14. Opie LH. The heart. Physiology and metabolism. New York: Raven Press, 1991.

15. Courtney KR, Sokolove PG. Importance of electrogenic sodium pump in normal and overdriven sinoatrial pacemaker. J Mol Cell Cardiol 1979; 11 : 787-94.

16. Difrancesco D, Camm JA. Heart rate lowering by specific and selective I(f) current inhibition with ivabradine: a new therapeutic perspective in cardiovascular disease. Drugs $2004 ; 64$ : 1757-65.
TIRÉS À PART

G. Lerebours

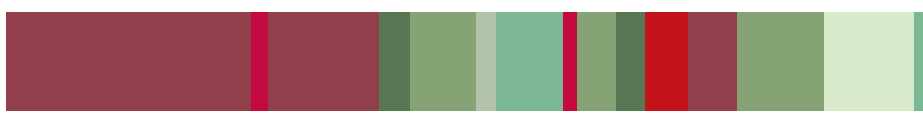

